\title{
REVIEW
}

\section{Study on Antitumor Activity and Mechanism of Compound Polysaccharide}

\author{
Man Li ( $^{*}$ \\ Department of Oncology, the Second Hospital of Dalian Medical University, Dalian 116027, Liaoning, China.
}

Corresponding Author: Man Li, Department of Oncology, the Second Hospital of Dalian Medical University, No. 467 Zhongshan Road, Shahekou Area, Dalian 116027, Liaoning, China. E-mail: Liman968@163.com

Abstract

In recent years, malignant tumors have become the number one major disease that seriously endangers health and causes death. The main methods for the treatment of malignant tumors are surgery, radiotherapy and chemotherapy. However, radiation therapy and chemotherapy have the disadvantages of poor specificity and damage to the immune function of the body. Therefore, the preparation of anti-cancer active ingredients with low toxicity and good efficacy can protect and improve the immune function of tumor patients. In particular, it is of great significance to improve the cellular immune function of patients and to reduce the toxic and side effects of radiotherapy and chemotherapy for preventing the occurrence of tumors and improving the therapeutic effect of tumors. A large number of studies have shown that polysaccharides can enhance immune function and anti-tumor effects. Plant polysaccharides from different sources have different mechanisms. Different polysaccharides are formulated into a mixed preparation according to a certain ratio. After entering the body, the anti-tumor and anti-radiation effects of the body will be improved through different approaches, and the mechanism of action will be explored, which will provide important experimental basis for clinical treatment of tumor patients.

Keywords

Compound polysaccharides; ginseng polysaccharides; pine mushroom polysaccharides; lentinan polysaccharides; immune regulation; cancer treatment; radiation protection

\section{INTRODUCTION}

Tumor is an abnormal pathological change in the expression of genes in local tissue cells due to carcinogenic factors. It cannot regulate the growth of cells normally, resulting in abnormal lesions caused by abnormal cell proliferation. Tumor can be divided into benign tumors and malignant tumors. The former does not recur or metastasize in general, and only blocks and squeezes tissues and organs. It can be cleaned up by surgical treatment. The latter is also called cancer. Cancer cells not only grow out of control, but also invade the surroundings without restriction. Normal tissue is transferred to other tissues of the body via the circulatory system. Due to the invasion of cancer cells, pain, bleeding, necrosis, infection, wasting, weakness, fever, and severe organ failure in the corresponding parts eventually lead to death ${ }^{[1-4]}$. According to statistics from the United Nations, one in eight deaths worldwide currently results from malignant tumors, which is much higher than the total number of

(C) The Author(s) 2019. Open Access This article is under the terms of Creative Commons Attribution 4.0 International License (https://creativecommons.org/licenses/by/4.0/), which permits unrestricted use, sharing, adaptation, distribution and reproduction in any medium or format, as long as you give appropriate credit to the original author(s) and the source, provide a link to the Creative Commons license, and indicate if changes were made. 
deaths caused by tuberculosis, Acquired Immune Deficiency Syndrome (AIDS) and malaria. Malignant tumors have become the first major disease that seriously endangers human health and causes human death ${ }^{[5]}$.

According to the World Health Organization (WHO) statistics report, the number of malignant tumors continues to increase at an average annual rate of 5\%. In 2012, there were about 14 million new malignant tumor patients worldwide and 8.2 million deaths. It is expected that by 2020, the number of new cases of malignant tumors will reach 15 million, and the number of deaths will reach 10 million $^{[6-10]}$. According to a report published by the American Cancer Society (ACS), the incidence of malignant tumors is increasing due to the aging of the population, unhealthy lifestyles and various environmental factors ${ }^{[11-12]}$. In recent years, the morbidity of malignant tumors in China have also shown a clear upward trend. About 3.5 million new cancer cases and about 2.5 million deaths due to cancer occur every year. According to the Global Cancer Report published by the WHO in 2014, China ranks first in the world for new cases and deaths in liver, esophagus, stomach, and lung malignancies. Malignant tumors cause great harm to human health, and bring a heavy economic and social burden to the world, especially developing countries. Therefore, how to effectively prevent and treat cancer is one of the important topics of concern in the medical community around the world.

\section{EXPERIMENTAL MATERIALS}

\subsection{Cell Line}

Mouse melanoma cells (B16) and mouse mast cell tumor cells (P815) were donated by the Basic Medical College of Jilin University. Mouse liver cancer cells (H22) and mouse lymphoma cells (YAC-1) were donated by the China-Japan Friendship Hospital of Jilin University.

\subsection{Experimental Animal}

Healthy male C57BL/6 mice of weighing (20 \pm 2$) \mathrm{g}$ at 6-8 weeks of age were were purchased from the Animal Experiment Center of Jilin University. Clean-grade healthy Institute of Cancer Research (ICR) mice of weighing (20 \pm 2 ) g at 6-8 weeks of age were purchased from Animal Experiment Center of Jilin University. The number of female and male mice is equal.

\subsection{Main Reagent}

RPMI-1640 culture medium, American GIBCO company; calf serum, Hangzhou Sijiqing Biological Engineering Co., Ltd.; trypsin, American Amresco company; Methylthiazoletrazolium (MTT), American Sigma company; ConA, Sigma, USA; DMSO, Sigma, USA; Potassium dihydrogen phosphate, Sinopharm Chemical Reagent Co., Ltd.; Disodium hydrogen phosphate, Sinopharm Chemical Reagent Co., Ltd.; Sodium chloride, Beijing Beihua Fine Chemicals Co., Ltd.; potassium chloride, Beijing Beihua Fine Chemicals Co., Ltd.; ammonium chloride, Sinopharm Chemical Reagent Co., Ltd.; Streptomycin sulfate, Dalian Meiluo Pharmaceutical Factory; Penicillin, North China Pharmaceutical Co., Ltd.; Methanol, Sinopharm Chemical Reagent Co., Ltd.; Glycerin, Sinopharm Chemical Reagent Co., Ltd.; Giemsa Staining Solution, Beijing Dingguo Biotechnology Co., Ltd.

\subsection{Main Instrument}

$\mathrm{CO}_{2}$ cell incubator, Thermo, USA; inverted microscope, Olympus, Japan; thermostatic water bath, Beijing Medical Equipment Factory; ultra clean bench, Suzhou purification equipment company; precision electronic balance, Sartorius, Germany; ultra-low temperature refrigerator, Thermo, USA; Ordinary refrigerator, China Haier Corporation; Constant temperature magnetic stirrer, Hangzhou Instrument Motor Factory; Microplate reader, American BIO-RAD; UV-visible spectrophotometer, Shanghai Daojinjin International Trade Co., Ltd.; Flow cytometer, American BD company; Ice machine, Ningbo Xinzhi Biotechnology Co., Ltd.; Autoclave, SANYO, Japan; Micropipette, Eppendorf, Germany; Low-temperature high-speed centrifuge, Thermo, USA.

\subsection{Preparation of Reagents}

Preparation of PBS: $0.20 \mathrm{~g} \mathrm{KCl}, 8.00 \mathrm{~g} \mathrm{NaCl}, 0.20 \mathrm{~g} \mathrm{KH}_{2} \mathrm{PO}_{4}$ and $3.49 \mathrm{~g} \mathrm{Na}_{2} \mathrm{HPO}_{4} \cdot 12 \mathrm{H}_{2} \mathrm{O}$ were dissolved in $800 \mathrm{~mL}$ double-distilled water and mixed thoroughly. The $\mathrm{pH}$ was adjusted to 7.2-7.4. $1000 \mathrm{~mL}$ with double-distilled water was removed from the solution using $0.22 \mu \mathrm{m}$ sterile filter and stored at $4{ }^{\circ} \mathrm{C}$.

Preparation of RPMI 1640 culture solution: The culture solution was prepared strictly according to the instructions. $2.0 \mathrm{~g}$ of $\mathrm{NaHCO}_{3}$ and $105 \mathrm{U}$ of streptomycin sulfate and penicillin were added to RPMI 1640 powder (10.4 g) to dissolve in $800 \mathrm{~mL}$ of double distilled water. The mixed solution was thoroughly stirred at room temperature for $4 \mathrm{~h}$, and the $\mathrm{pH}$ was adjusted to 7.2. The volume was fixed to $1000 \mathrm{~mL}$ with double steamed water. The bacteria in the solution were removed with a sterile filter membrane of $0.22{ }^{\circ} \mathrm{C}$ and stored at $4{ }^{\circ} \mathrm{C}$. 
Trypsin preparation: $0.5 \mathrm{~g}$ of trypsin powder was added to $200 \mathrm{~mL}$ of PBS. After the solution was fully dissolved, the bacteria in the solution were removed with a sterile filter membrane of $0.22 \mu \mathrm{m}$, and then stored in sterile vials at $-20{ }^{\circ} \mathrm{C}$.

Preparation of MTT: $0.5 \mathrm{~g}$ MTT was dissolved in $100 \mathrm{~mL}$ PBS to form a solution with a concentration of $5 \mathrm{mg} / \mathrm{mL}$. The bacteria in the solution were removed with a sterile filter membrane of $0.22 \mu \mathrm{m}$ and stored at $4{ }^{\circ} \mathrm{C}$ away from light.

Preparation of Giemsa stock solution: $3.8 \mathrm{~g}$ Giemsa dye powder and a small amount of methanol were ground in a mortar. 375 $\mathrm{mL}$ of methanol and $125 \mathrm{~mL}$ of glycerin were added, mixed thoroughly and placed in a $37^{\circ} \mathrm{C}$ incubator for 48 hours. It should be shaken several times during this period to fully dissolve the dye. The solution is then filtered and left for two weeks before use.

\section{EXPERIMENTAL METHOD}

\subsection{Antitumor Effect of Polysaccharides in vitro}

B16, H22, P815 and YAC-1 cells were treated with RPMI-1640 medium containing 10\% fetal bovine serum (FBS), and cells in logarithmic growth phase were used for subsequent experiments. Lentinus edodes and matsutake were purchased from Yichun City, Heilongjiang Province, and ginseng was purchased from Changbai Mountain, Jilin Province. Polysaccharides from lentinus edodes (PLE), polysaccharides from tricholoma matsutake (PTM) and polysaccharides from ginseng (PG) were extracted, purified, and purified in the department of health inspection, school of public health, Jilin University. The three polysaccharides were identified.

MTT assay was used to detect the inhibitory effect of PLE, PTM and PG on the growth of B16 and H22 cells. Cells in logarithmic growth phase were taken, and the density of each cell was adjusted to $5 \times 10^{4} \mathrm{~mL}$ to add to a 96 -well plate with 200 $\mu \mathrm{l}$ cell suspension per well. When the cell confluence reaches about $80 \%$, three kinds of polysaccharides are added respectively, and the final concentrations are 0, 50, 100, 200, 300, 400, and $500 \mu \mathrm{g} / \mathrm{mL}$. Each concentration is set in three replicates and placed at $37{ }^{\circ} \mathrm{C}$. In a $\mathrm{CO}_{2}$ incubator, the solution was cultured at different time gradients for $24 \mathrm{~h}, 48 \mathrm{~h}$, and $72 \mathrm{~h}$. Four hours before the end of the culture, $20 \mu \mathrm{l}$ of MTT solution $(5 \mathrm{mg} / \mathrm{mL})$ was added to each well. After $4 \mathrm{~h}$ of reaction, the supernatant was discarded, $150 \mu \mathrm{l}$ of DMSO was added, and the pellet was shaken to dissolve for $10 \mathrm{~min}$. The wavelength of $490 \mathrm{~nm}$ was detected using a microplate reader. The absorbance value is calculated and the cell survival rate is calculated.

Based on the effects of lentinan, matsutake polysaccharide and ginseng polysaccharide on the cytotoxicity of cytotoxic T lymphocyte (CTL), the L9 (34) orthogonal experiment was used to study the compound formula of three polysaccharides. Three factors are lentinus edodes (A), polysaccharides from tricholoma matsutake (B) and polysaccharides from ginseng (C). According to the experimental results, three levels are selected for each factor. The compound polysaccharide was used as the test object, and the experiment was performed according to the experimental method. The CTL cytotoxicity was calculated, and the best combination was selected. Table 1 shows the levels of polysaccharides by orthogonal test.

Table 1 The levels of polysaccharides by orthogonal test table $(\mu \mathrm{g} / \mathrm{mL})$

\begin{tabular}{cccc}
\hline Levels & A (PLE) & B (PLE) & C (PG) \\
\hline 1 & 50 & 50 & 50 \\
2 & 100 & 100 & 100 \\
3 & 200 & 200 & 200 \\
\hline
\end{tabular}

Note: PLE: polysaccharides from lentinus edodes; PG: polysaccharides from ginseng

The composition and dose of complex polysaccharides were determined based on the results of orthogonal experiments. The in vitro experiments were divided into four groups: blank control group, complex polysaccharide low dose group, complex polysaccharide medium dose group and complex polysaccharide high dose group. The compound polysaccharide low-dose group was administered at a dose of $300 \mu \mathrm{g} / \mathrm{mL}$ (including $100 \mu \mathrm{g} / \mathrm{mL}$ lentinan, $100 \mu \mathrm{g} / \mathrm{mL}$ pine mushroom polysaccharide, and $100 \mu \mathrm{g} / \mathrm{mL}$ ginseng polysaccharide). The compound polysaccharide medium dose group was administered with a dose of $600 \mu \mathrm{g} / \mathrm{mL}$ (including $200 \mu \mathrm{g} / \mathrm{mL}$ lentinan, $200 \mu \mathrm{g} / \mathrm{mL}$ matsutake polysaccharide, and $200 \mu \mathrm{g} / \mathrm{mL}$ ginseng polysaccharide). The compound polysaccharide high dose group was administered with a dose of $1200 \mu \mathrm{g} / \mathrm{mL}$ (including $400 \mu \mathrm{g} / \mathrm{mL}$ lentinan, 400 $\mu \mathrm{g} / \mathrm{mL}$ matsutake polysaccharide, and $400 \mu \mathrm{g} / \mathrm{mL}$ ginseng polysaccharide).

3.2 Inhibitory Effect of Complex Polysaccharides and Chemotherapy Drugs on Tumor Growth in B16 TumorBearing Mice

B16, P815 and YAC-1 cells were treated with RPMI-1640 medium containing 10\% FBS, and cells in logarithmic growth phase 
were used for subsequent experiments. B16 cells were grown adherently in RPMI-1640 medium containing 10\% FBS. When the cell growth is in the logarithmic phase, the cells were collected, the number of cells was counted, and the concentration of the cells was adjusted to $1 \times 10^{6}$ cells $/ \mathrm{mL}$ with sterile saline. The inoculation dose was $2 \times 10^{5}$ cells $/$ mouse, and each mouse was treated similarly. Eighty ICR mice inoculated with B16 tumors were randomly divided into 8 groups with 10 in each group, and another 10 healthy mice were set as blank control groups. The mice in each group were given corresponding treatment factors $24 \mathrm{~h}$ after tumor inoculation.

After the mice were sacrificed by dislocation, the sternum was removed, and the muscles around the sternum were discarded. The blood stain was wiped out, and the bone marrow fluid was squeezed out with a hemostat. The bone marrow fluid was dropped into calf serum at one end of the slide (the end prepared in advance had slides of calf serum droplets), and mixed thoroughly. The fluid was smeared according to the routine blood smear method, dried naturally in the air, and fixed in methanol for $10 \mathrm{~min}$. Then, the slide was removed. After drying, it was put in Giemsa application solution, stained for 10-15 minutes, and rinsed with distilled water. The obtained object was dried and the number of micronucleus cells in 1000 polychromatic erythrocytes in each animal was counted under the microscope.

\section{RESULTS}

Mushroom polysaccharides, pine mushroom polysaccharides and ginseng polysaccharides were co-cultured with tumor cells B16 and H22 cells in vitro. MTT assay showed that lentinan and ginseng polysaccharides had a certain inhibitory effect on the growth of B16 and $\mathrm{H} 22$ cells, but the inhibition rates did not exceed 15\%. It has no significant killing effect on tumor cells. When the concentration is $200 \mu \mathrm{g} / \mathrm{mL}$, the inhibition rate of B16 and $\mathrm{H} 22$ cells is the largest when it is incubated for $48 \mathrm{~h}$.

As the polysaccharide concentration increased, the lymphocyte stimulation index of each polysaccharide increased. When the PLE concentration was $300 \mu \mathrm{g} / \mathrm{mL}$, the lymphocyte proliferation ability was the strongest, and when the PTM concentration was $200 \mu \mathrm{g} / \mathrm{mL}$, the lymphocyte proliferation ability was the strongest. When the PG concentration was $200 \mu \mathrm{g} / \mathrm{mL}$, the lymphocyte proliferation ability was the strongest. As the incubation time of lentinan and matsutake polysaccharide increased, the corresponding lymphocyte stimulation index increased. Increasing the incubation time of ginseng polysaccharide had no significant effect on the change of lymphocyte stimulation index.

As the concentration of lentinan increased, the killing activity of CTL cells increased. When the concentration was greater than 300 $\mu \mathrm{g} / \mathrm{mL}$, the change became gentle. When the concentration of Tricholoma matsutake polysaccharide was $200 \mu \mathrm{g} / \mathrm{mL}$, the killing activity of CTL cells was the largest. When the concentration of ginseng polysaccharide was $100 \mu \mathrm{g} / \mathrm{mL}$, the killing activity of CTL cells was the largest. As the concentration of ginseng polysaccharide increased, the killing effect of CTL cells first increased and then decreased.

The value of three polysaccharides on the cytotoxic activity can be obtained from the correlation coefficient. The order is matsutake polysaccharides $>$ ginseng polysaccharides $>$ lentinan polysaccharides. Table 2 shows the experiment results of three kinds of polysaccharide complex formula orthogonal. According to Table 2, the best combination of the three polysaccharides is A3B3C3. Lentinan polysaccharides: Matsutake polysaccharides: Ginseng polysaccharide = 1: 1: 1 .

Table 2 Three kinds of polysaccharide complex formula orthogonal experiment results

\begin{tabular}{ccccc}
\hline Number & A (PLE) & B (PTM) & C (PG) & CTL $(\%)$ \\
\hline 1 & 1 & 1 & 1 & 22.425 \\
2 & 1 & 2 & 2 & 29.596 \\
3 & 1 & 3 & 3 & 43.937 \\
4 & 2 & 1 & 2 & 33.246 \\
5 & 2 & 2 & 3 & 35.332 \\
6 & 2 & 3 & 1 & 38.462 \\
7 & 3 & 1 & 3 & 43.677 \\
8 & 3 & 2 & 1 & 29.857 \\
9 & 3 & 3 & 2 & 48.631 \\
K1 & 31.986 & 33.116 & 30.248 & \\
K2 & 35.68 & 31.595 & 37.158 & 40.982 \\
K3 & 40.722 & 43.677 & 10.734 & \\
R & 8.736 & 12.082 & & \\
\hline
\end{tabular}

Note: PLE: polysaccharides from lentinus edodes; PTM: polysaccharides from tricholoma matsutake; PG: polysaccharides from ginseng; CTL: cytotoxic T lymphocyte 


\section{DISCUSSION}

Lentinus edodes and matsutake are valuable edible and medicinal fungi in China, which are rich in nutrition and delicious in taste. The polysaccharides they contain have good anti-tumor effects. Ginseng has been considered as a longevity supplement. Its medicinal value has been recognized by the world. Polysaccharides are the active ingredients of ginseng, which have anti-tumor, anti-oxidation and immune regulation effects. Although many studies report the biological activities of lentinan, matsutake, and ginseng polysaccharides, there is no systematic research on them. Polysaccharides are a class of natural macromolecules. The antitumor mechanism of polysaccharides from different sources is also different. The antitumor mechanism of the three polysaccharides selected in this project is as follows:

Polysaccharides mainly inhibit tumor growth through two ways. One is to improve the immune function of the host, regulate the level of cytokines, and exert antitumor effects. The other way is that polysaccharides can directly inhibit the growth of tumor cells. Ginseng polysaccharide has a wide range of biological activities. In addition, it has a significant promotion effect on the body's immune function, and promotes the increase of the spleen and thymus index. The spleen and thymus are important central immune organs of the body. It contains a large number of immune cells, which can limit the occurrence and development of tumors through a variety of mechanisms. The combined application of ginseng polysaccharides and chemotherapeutic drugs can significantly increase the spleen and thymus index decline caused by chemotherapeutic drugs. Ginseng polysaccharides can also induce spleen cells to produce a variety of anti-cancer factors. GM-CSF is an important regulator of hematopoietic cell proliferation and differentiation. It can stimulate the proliferation of a variety of immune cells, such as T cells, macrophages, neutrophils, eosinophils, pluripotent stem cells, and megakaryocytes. Leukopenia caused by tumor chemoradiotherapy wasa relieved and complications such as infection were prevented. TNF- $\alpha$ can not only regulate the body's immune function, but also inhibit the formation of neovascularization in tumor tissues and induce tumor cells to undergo apoptosis. IL-2 is secreted by $\mathrm{T}$ cells, which can stimulate natural killer (NK) cell proliferation, induce lymphokine activated killer (LAK) cell production, and activate macrophages. Interferon- $\gamma(\mathrm{IFN}-\gamma)$ can induce macrophages to produce nitric oxide synthase, and promote the synthesis of the signaling molecule. Signaling molecule can resist foreign toxicants invading the body and prevent the proliferation and spread of tumor cells. Ginseng polysaccharide can promote both specific and non-specific immunity of the body, increase the activity of NK and CTL cells, promote lymphocyte proliferation, enhance the phagocytosis of macrophages, and induce macrophages to produce signaling molecule, IL-12, IL cytokines such as -6 . TNF- $\alpha$ can restore the reduced activity of NK and CTL cells caused by chemotherapy drugs, enhance the binding activity of complement, and play an anti-tumor role.

Dendritic cell (DC) plays an important role in specific cellular immune response. It is the initiator of the body's immune response. DC can take up antigens and play an important role in the body's immune regulation. It is the most powerful antigenpresenting cell and can inhibit tumorigenesis. Studies have reported that ginseng polysaccharides can promote the secretion of cytokines with strong anticancer activity such as TNF- $\alpha$, IL-12 and IL-2 by DC, stimulate the expression of DC surface molecules, and inhibit the growth of tumor cells. Ginseng polysaccharide can inhibit the proliferation of a variety of tumor cells by regulating the cell division cycle of tumors, activating tumor regulation-related signal transduction molecules, and enhancing the expression of anti-oncogenes. Ginseng polysaccharide can enhance the expression of P21 cyclin, stop tumor cell division at the G0/GI or G2/M phase of the cell cycle, and also activate the pathway to enhance the body's immune function. P53 gene is an anti-cancer gene. Ginseng polysaccharide can enhance the expression of P53 gene in S180 tumor-bearing mice and inhibit the growth of S180 tumor cells. Ginseng polysaccharide can inhibit the proliferation of B16-F10 tumor cells by inducing the expression of P53 and P21 genes and activating the expression of caspase- 8 protein. Acidic ginseng polysaccharides can inhibit the proliferation of primary lung cancer cells induced by toxicants, and can also inhibit the metastasis of lung metastatic B16-F10 tumors in the lung. When used in combination with recombinant IL-2, it can inhibit the growth of lung cancer cells and recombine with the combined application of IFN- $\gamma$, enhance the phagocytic function of macrophages and improve the killing activity of cytotoxic T lymphocytes on tumor cells. When combined with paclitaxel, it can antagonize the secretion of paclitaxel to the body by enhancing cell activity and stimulating IL-6 secretion, and enhance the antitumor effect of paclitaxel.

The combined application of ginseng polysaccharides and small molecule anti-tumor substances can reduce the toxicity of small molecule anti-tumor substances while enhancing the anti-tumor efficacy. Researchers have shown that ginseng 
polysaccharides have no significant inhibitory effect on Ehrlich, S180, and tumor cell growth in vitro, but it can significantly inhibit the growth of lung cancer 3LL solid tumors, adenocarcinoma JC, Ehrlich, and S180 ascites tumor cells in vivo and enhance the immune function. The experimental results show that ginseng polysaccharide has no direct killing effect on B16 and H22 tumor cells in vitro, but it can significantly enhance the killing activity of CTL on P815 tumor cells.

\section{CONCLUSION}

In this study, three polysaccharides extracted from Trichotomy matsutake, Lentinula erodes and ginseng were used to make composite polysaccharides at a certain ratio to detect the inhibitory effect of composite polysaccharides on the growth of B16 and H22 tumor cells and the antitumor effect of combined use with chemotherapy drugs. In this study, we found that compound polysaccharides can enhance the effect of chemotherapeutic drugs on the inhibition of B16 and $\mathrm{H} 22$ tumor growth. Compared with the effects of chemotherapeutic drugs alone, the combined use of compound polysaccharides and chemotherapeutic drugs can significantly reduce the tumor weight and volume of tumor-bearing mice.

In summary, a compound polysaccharide composed of polysaccharides from velvet mushroom, lentinan, and ginseng can enhance the therapeutic effect of chemotherapeutic drugs, antagonize the damage of chemotherapeutic drugs to the body's immune system, and inhibit the growth of tumors. The effect of compound polysaccharide adjuvant chemotherapy drugs on tumor cell growth is significantly higher than that of a single polysaccharide.

\section{REFERENCE}

[1] Ren L, Perera C, Hemar Y. Antitumor activity of mushroom polysaccharides: a review. Food Funct 2012,3(11),1118-1130.

[2] Mizuno M, Nishitani Y. Immunomodulating compounds in Basidiomycetes. J Clin Biochem Nutr 2013,52(3),202-207.

[3] Daba AS, Ezeronye OU. Anti-cancer effect of polysaccharides isolated from higher basidiomycetes mushrooms. Afr J Biotechnol 2003,2(12),672-678.

[4] Zhang M, Cui SW, Cheung PCK, Wang Q. Antitumor polysaccharides from mushrooms: a review on their isolation process, structural characteristics and antitumor activity. Trends Food Sci Technol 2007,18(1),4-19.

[5] Ferreira IC, Heleno SA, Reis FS, Stojkovic D, Queiroz MJ, Vasconcelos MH, Sokovic M. Chemical features of Ganoderma polysaccharides with antioxidant, antitumor and antimicrobial activities. Phytochem 2015,114,38-55.

[6] Zhao L, Dong Y, Chen G, Hu Q. Extraction, purification, characterization and antitumor activity of polysaccharides from Ganoderma lucidum. Carbohydr Polym 2010,80(3),783-789.

[7] Pandya U, Dhuldhaj U, Sahay NS. Bioactive mushroom polysaccharides as antitumor: an overview. Nat Prod Res 2019,33(18),2668-2680.

[8] Song G, Du Q. Structure characterization and antitumor activity of an $\alpha \beta$-glucan polysaccharide from Auricularia polytricha. Int Food Res J 2012,45(1),381-387.

[9] Chatterjee S, Biswas G, Basu SK, Acharya K. Antineoplastic effect of mushrooms: a review. Aust J Crop Sci 2011,5(7), 904.

[10] Shang D, Cui Q, Li Y, Yu Z, Wen L, Zhao Y, Zhang J. Antitumor mechanism of Se-containing polysaccharide, a novel organic selenium compound. Front Biol 2009,4(3),248-253.

[11] Saima Y, Das AK, Sarkar KK, Sen Sr AK, Sur P. An antitumor pectic polysaccharide from Feronia limonia. Int J Biol Macromol 2000,27(5),333-335.

[12] Xu AH, Chen HS, Sun BC, Xiang XR, Chu YF, Zhai F, Jia LC. Therapeutic mechanism of ginkgo biloba exocarp polysaccharides on gastric cancer. World J Gastroenterol 2003,9(11),2424. 